\title{
Challenges Staging Neuroendocrine Tumors of the Pancreas, Jejunum and Ileum, and Appendix
}

\author{
Eric K. Nakakura, MD, PhD
}

Division of General Surgery, Section of Surgical Oncology/Hepatopancreaticobiliary Surgery, Department of Surgery and Helen Diller Family Comprehensive Cancer Center, University of California, San Francisco, CA

Neuroendocrine tumors (NETs) comprise a heterogeneous group of neoplasms, making it a real challenge to devise practical staging systems, which provide accurate prognostic information. For example, NETs of the pancreas, small intestine, and appendix are highly disparate. Moreover, within each organ site, NETs have greatly varying biology and natural history: duodenal NETs differ from jejunal/ileum NETs; insulinomas differ from other functional and nonfunctional pancreatic NETs. ${ }^{1}$ Even within a particular stage of a primary site, natural history can vary greatly. Patients with stage IV NETs of the jejunum/ileum who have oligometastatic disease in the liver do much better than those who have diffuse bilobar liver or extensive peritoneal metastases. ${ }^{2}$

Notwithstanding these challenges, the AJCC staging system organizes the anatomic extent of disease into defined stage groupings with prognostic value. Here, changes in the 8th edition of the AJCC staging system for NETs of the pancreas, jejunum/ileum, and appendix are highlighted. ${ }^{3}$ In addition, practical aspects of intraoperative staging unique to NETs are discussed.

\section{NEUROENDOCRINE TUMORS OF THE PANCREAS}

A major change in the 8th edition of the AJCC staging system for NETs of the pancreas (PNETs) is that well-differentiated PNETs are staged using a new TNM staging system and are no longer lumped with exocrine pancreas

(C) Society of Surgical Oncology 2017

First Received: 20 June 2017;

Published Online: 4 August 2017

E. K. Nakakura, MD, PhD

e-mail: Eric.Nakakura@ucsf.edu neoplasms. ${ }^{3}$ It may not be surprising that well-differentiated PNETs and exocrine pancreas neoplasms each need their own unique TNM staging system given their disparate biology and natural history. For example, well-differentiated PNETs are slow growing, and primary tumors are well circumscribed, infrequently invading adjacent organs and regional vessels (celiac axis, superior mesenteric artery) only when large. In contrast, pancreas ductal adenocarcinomas are more aggressive, characterized by rapid growth, early metastasis to regional lymph nodes and distant sites, and frequent invasion of adjacent organs and vessels.

The 8th edition AJCC staging system is based on the European Neuroendocrine Tumor Society TNM staging system (ENETS TNM), which was developed in 2006. ${ }^{1}$ The AJCC staging system applies to well-differentiated PNETs, which are characterized by a low-intermediate proliferative and mitotic index $(\mathrm{G} 1, \mathrm{G} 2)$. It also applies to PNETs with well-differentiated histology, which on occasion can have a higher proliferative index (G3). ${ }^{4}$ However, G3 typically describes a poorly differentiated neuroendocrine carcinoma, which is staged like exocrine pancreas neoplasms. In the most recent and largest study evaluating patients $(N=1072)$ who underwent surgery for PNETs, Rindi et al. showed that the ENETS TNM was better than the 7th edition AJCC staging system, especially when it is simplified into four stage groups (I, II, III, IV). ${ }^{1,5}$ The ENETs TNM is based mainly on size and does not include peripancreatic soft-tissue invasion.

An important point to emphasize for surgeons and pathologists is that Rindi et al. found that a single lymph node metastasis was significantly associated with adverse survival. However, only 335 of 1072 (31\%) patients had lymph nodes assessed. ${ }^{1}$ That is, lymph nodes were not sampled or data were not available for $\sim 70 \%$ PNET resection specimens in their study. Other smaller, single-institutional studies have reported that lymph nodes are not sampled in 
30-37\% PNET resection specimens. ${ }^{6}$ Because lymph node metastasis is a poor prognostic factor for PNETs, it is essential that lymph nodes are sampled adequately.

What are the reasons for the low lymph node sampling rate in PNET resection specimens? Enucleation and spleen-preserving distal pancreatectomy are both associated with zero or few lymph nodes sampled and should be reserved for insulinomas, which are typically benign. ${ }^{6}$ However, other functioning (i.e., noninsulinoma) and nonfunctioning PNETs have a more aggressive course and should be managed with oncologic resections of the primary tumor and regional lymph nodes. ${ }^{1}$ One exception may be small $(<1-2 \mathrm{~cm})$, nonfunctioning PNETs in the elderly or those with comorbid conditions, where observation may be more appropriate. ${ }^{7,8}$ When surgery is performed, surgeons and pathologists need to ensure that optimal lymph node sampling is done for PNETs, except for insulinomas, so patients can be accurately staged. ${ }^{6}$

\section{NEUROENDOCRINE TUMORS OF THE JEJUNUM AND ILEUM}

In the 8th edition AJCC staging system, the prior chapter on NETs of the small bowel has been separated into a chapter on jejunum/ileum NETs and a chapter combining duodenum and ampulla of Vater NETs. ${ }^{3}$ Thus, the new chapter on NETs of the jejunum and ileum pertains to the "classic" small bowel neuroendocrine, or carcinoid, tumor, which is primarily a NET of the ileum, and less commonly, the jejunum. Tumors of the ileum frequently spread to regional lymph nodes, causing fibrosis of the mesentery (mesenteric mass), which often is the only sign of the tumor on imaging because primary tumors are too small to be seen. ${ }^{9}$ When NETs of the ileum spread to the liver or ovaries, patients may develop the carcinoid syndrome.

Therefore, surgeons must perform systematic intraoperative staging. In $20 \%$ of patients, peritoneal metastases are identified. ${ }^{10}$ Up to $61 \%$ of patients have liver metastases, so the liver surface should be carefully evaluated, and intraoperative ultrasound can be done to detect small intrahepatic lesions. ${ }^{10}$ In $4 \%$ of patients, ovarian metastases are present, so both ovaries should be examined. ${ }^{10}$ Because primary tumors are typically very small, the entire jejunum and ileum (from the ligament of Treitz to the ileocecal valve) must be carefully palpated. ${ }^{9}$ Because primary tumors are frequently (in 25-44\% of patients) multifocal and multifocal tumors often are very small $(<1 \mathrm{~cm})$, they can only be found by meticulous palpation. ${ }^{2}$

Importantly, if surgeons are performing laparoscopic or minimally invasive surgery, the entire jejunum and ileum should be palpated digitally to identify small primary tumors that are frequently multifocal. ${ }^{2,9,11,12}$ This can be done using a hand-assisted laparoscopic device (Gelport;
Applied Medical) or a soft-tissue wound retractor (Alexis Wound Retractor; Applied Medical) to exteriorize the jejunum and ileum. ${ }^{9,12}$ With the jejunum and ileum outside the abdomen, complete palpation, resection of the mesenteric adenopathy/fibrosis and primary tumor(s), and intestinal anastomosis can be done. ${ }^{9,12}$

In $\sim 13 \%$ of patients diagnosed with NETs, the primary site is not known. ${ }^{12}$ For well-differentiated NETs of unknown primary, recent studies suggest that most can be found by surgical exploration, and most are located in the ileum. ${ }^{12}$ The reason is that NETs of the ileum are small, located in the submucosa, and have an outward growth pattern, so it is very difficult to detect them with endoscopy or cross sectional imaging. ${ }^{9,12,13}$ Again, digital palpation is critical to identify them. ${ }^{2}$

For NETs of the jejunum and ileum, the AJCC TNM staging classification imperfectly provides prognostic information. ${ }^{3}$ Death rates remain stable from stage I to III and only significantly differ between stage III and IV. ${ }^{3}$ Outcome analyses are confounded by the indolent course of locoregional disease (stage I-III), limited follow-up times, few patients with local disease (stage I-II), most patients with regional and distant disease (stage III-IV), and the heterogeneous nature of patients with stage III and stage IV disease. ${ }^{3,14}$ Importantly, pathologic staging requires evaluation of specimens obtained from surgery, and significant differences may exist regarding patient selection for surgery and surgical technique, such as extent of mesenteric dissection, management of liver metastases (i.e., major hepatectomy, enucleation, ablation).

The heterogeneous nature of stage IV disease was already mentioned. For patients with stage III disease (lymph node metastasis), the main cause of morbidity is the mesenteric fibrosis, resulting from the desmoplastic reaction of the NET lymph node metastases. The mesenteric fibrosis buckles the ileum/jejunum, causing small-bowel obstruction and impairs blood flow, resulting in ischemia. ${ }^{13}$ The goals of surgery are resection of the primary tumor(s), regional lymph nodes, and mesenteric fibrosis, if feasible, maximizing the length of viable intestine. ${ }^{2}$ However, large $(>2 \mathrm{~cm})$ mesenteric masses or encasement of the proximal superior mesenteric artery and vein may preclude safe resection. Another change to the 8th edition of the AJCC staging system is a new classification of nodal involvement, N2, so information on the prognostic significance of large $(>2 \mathrm{~cm})$ mesenteric masses or encasement of mesenteric vessels can be collected. ${ }^{3}$

\section{NEUROENDOCRINE TUMORS OF THE APPENDIX}

Most patients with appendiceal NETs are diagnosed after an appendectomy is done for presumed appendicitis 
and pathological examination reveals the diagnosis. A right hemicolectomy should be done for patients with a tumor $>2 \mathrm{~cm}$ or an incompletely resected tumor. ${ }^{15}$ In addition, a right hemicolectomy may be considered for patients with a 1 - to 2-cm tumor and the presence of lymphovascular invasion, mesoappendiceal involvement, or atypical histologic features. ${ }^{15}$ For NETs of the appendix, only a minor change was made for the 8th edition-an elimination of substages $\mathrm{A}$ and $\mathrm{B} .^{3}$

\section{CONCLUSIONS}

Surprisingly, the first TNM staging system for NETs was developed by ENETs in 2006, and the AJCC TNM staging system for NETs first appeared in the 7th edition in $2010 .{ }^{1,5}$ An examination of the $8^{\text {th }}$ edition of the AJCC TNM staging system for NETs of the pancreas, jejunum and ileum, and appendix highlights the challenges of developing classifications for diverse NETs that provide accurate prognostic discrimination and that are widely applicable. Only through the prospective collection of information will further refinements be possible, leading to improved prognostic validity and clinical applicability.

\section{REFERENCES}

1. Rindi G, Falconi M, Klersy C, Albarello L, Boninsegna L, Buchler MW, et al. TNM staging of neoplasms of the endocrine pancreas: results from a large international cohort study. $J$ Natl Cancer Inst. 2012;104(10):764-77. doi:10.1093/jnci/djs208.

2. Howe JR, Cardona K, Fraker DL, Kebebew E, Untch BR, Wang YZ, et al. The surgical management of small bowel neuroendocrine tumors: consensus guidelines of the North American Neuroendocrine Tumor Society. Pancreas. 2017;46(6):715-31. doi:10.1097/MPA.0000000000000846.

3. Amin MB, Edge SB, Greene FL, Byrd DR, Brookland RK, Washington MK, et al. (eds) AJCC cancer staging manual, 8th edn, New York: Springer; 2017.

4. Basturk O, Yang Z, Tang LH, Hruban RH, Adsay V, McCall CM, et al. The high-grade (WHO G3) pancreatic neuroendocrine tumor category is morphologically and biologically heterogenous and includes both well differentiated and poorly differentiated neoplasms. Am J Surg Pathol. 2015;39(5):683-90. doi:10.1097/ PAS.0000000000000408.

5. Edge SB, Byrd DR, Compton CC, Fritz AG, Greene FL, Trotti A (eds), 7th edn. Chicago: Springer; 2010. p. 183-7.

6. Parekh JR, Wang SC, Bergsland EK, Venook AP, Warren RS, $\mathrm{Kim} \mathrm{GE}$, et al. Lymph node sampling rates and predictors of nodal metastasis in pancreatic neuroendocrine tumor resections: the UCSF experience with 149 patients. Pancreas. 2012;41(6):840-4. doi:10.1097/MPA.0b013e31823cdaa0.

7. Crippa S, Partelli S, Zamboni G, Scarpa A, Tamburrino D, Bassi $\mathrm{C}$, et al. Incidental diagnosis as prognostic factor in different tumor stages of nonfunctioning pancreatic endocrine tumors. Surgery. 2014;155(1):145-53. doi:10.1016/j.surg.2013.08.002.

8. Toste PA, Kadera BE, Tatishchev SF, Dawson DW, Clerkin BM, Muthusamy R, et al. Nonfunctional pancreatic neuroendocrine tumors $<2 \mathrm{~cm}$ on preoperative imaging are associated with a low incidence of nodal metastasis and an excellent overall survival. $J$ Gastrointest Surg. 2013;17(12):2105-13. doi:10.1007/s11605013-2360-9.

9. Wang SC, Parekh JR, Zuraek MB, Venook AP, Bergsland EK, Warren RS, et al. Identification of unknown primary tumors in patients with neuroendocrine liver metastases. Arch Surg. 2010;145(3):276-80. doi:10.1001/archsurg.2010.10.

10. Norlén O, Stålberg P, Öberg K, Eriksson J, Hedberg J, Hessman $\mathrm{O}$, et al. Long-term results of surgery for small intestinal neuroendocrine tumors at a tertiary referral center. World J Surg. 2012;36(6):1419-31. doi:10.1007/s00268-011-1296-z.

11. Wang SC, Fidelman N, Nakakura EK. Management of well-differentiated gastrointestinal neuroendocrine tumors metastatic to the liver. Semin Oncol. 2013;40(1):69-74. doi:10.1053/j. seminoncol.2012.11.007.

12. Bergsland EK, Nakakura EK. Neuroendocrine tumors of unknown primary: is the primary site really not known? JAMA Surg. 2014;149(9):889-90. doi:10.1001/jamasurg.2014.216.

13. Moertel CG. Karnofsky memorial lecture. An odyssey in the land of small tumors. J Clin Oncol. 1987;5(10):1502-22.

14. Strosberg JR, Weber JM, Feldman M, Coppola D, Meredith K, Kvols LK. Prognostic validity of the American Joint Committee on Cancer staging classification for midgut neuroendocrine tumors. J Clin Oncol. 2013;31(4):420-25. doi:10.1200/JCO. 2012.44.5924.

15. www.nccn.org/professionals/physician_gls/pdf/neuroendocrine. pdf. 